\title{
DIVISION SEXUELLE DU TRAVAIL ET DOMINATION SOCIALE : RETOUR SUR QUELQUES IDÉES REÇUES
}

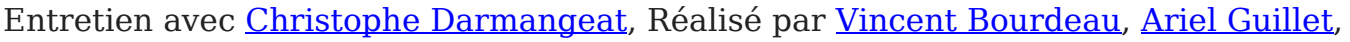
Chloé Santoro

\author{
La Découverte | «Mouvements »
}

2021/2 n 106 | pages 153 à 161

ISSN 1291-6412

ISBN 9782348069543

Article disponible en ligne à l'adresse :

https://www.cairn.info/revue-mouvements-2021-2-page-153.htm

Distribution électronique Cairn.info pour La Découverte.

(C) La Découverte. Tous droits réservés pour tous pays.

La reproduction ou représentation de cet article, notamment par photocopie, n'est autorisée que dans les limites des conditions générales d'utilisation du site ou, le cas échéant, des conditions générales de la licence souscrite par votre établissement. Toute autre reproduction ou représentation, en tout ou partie, sous quelque forme et de quelque manière que ce soit, est interdite sauf accord préalable et écrit de l'éditeur, en dehors des cas prévus par la législation en vigueur en France. Il est précisé que son stockage dans une base de données est également interdit. 


\section{Division sexuelle du travail et domination sociale : \\ retour sur quelques idées reçues}

Entretien avec Christophe Darmangeat

Christophe Darmangeat réfléchit depuis de nombreuses années à la question de la division sexuelle du travail dans les sociétés préétatiques. Auteur de Le Communisme primitif n'est plus ce qu'il était. Aux origines de l'oppression des femmes (Smolny, 2012), il n'a cessé d'interroger la manière dont les sociétés organisent le travail et ses conséquences en termes d'oppression ou d'exploitation.
Mouvements (Mvts): Partons d'une question simple: comment définiriez-vous la division sexuelle du travail? Peut-on dire que c'est un trait structurant de toute société (ancienne, moderne, occidentale, extra-européenne)?

Christophe Darmangeat (C. D.) : La division sexuelle (ou sexuée) du travail consiste en une répartition plus ou moins systématique et plus ou moins prescriptive des tâches productives selon le sexe des individus, doublée d'une obligation de mettre une partie de son produit à disposition de l'autre, en particulier au sein de la famille hétérosexuelle lorsque cette configuration des relations d'alliance et de filiation existe.

Ainsi, très classiquement, chez les peuples dépourvus d'agriculture et d'élevage - même si c'est un cliché, il repose sur une réalité -, les hommes chassent et donnent une partie de leurs prises aux femmes, qui ramassent des végétaux et donnent une partie de leur cueillette aux hommes. D'une société à l'autre, cette répartition des tâches peut connaître certaines variations ; de même que la mise en commun des produits, elle peut être plus ou moins stricte et systématique, mais on ne connaît aucun exemple où elle serait absente. De ce point de vue, le modèle contemporain dit de "l'égalité des sexes " constitue une rupture avec tout ce que l'on connaît
RÉALISÉ PAR

VINCENT

BOURDEAU ${ }^{1}$, Ariel Guillet ${ }^{2}$ et Chló Santoro 3

1. Membre du comité de rédaction de Mouvements.

2. Doctorant en philosophie, Logiques de l'Agir (E.A.

2274), Université de Besançon.

3. Doctorante en philosophie, Logiques de l'Agir (E.A. 2274), Université de Besançon. 
du passé de l'humanité. Nous vivons en effet dans la première société qui ait aboli l'ensemble des interdits normatifs, notamment professionnels, en fonction du sexe et qui, au moins en paroles, proclame qu'en plus de disparaître de la norme, la division sexuelle du travail devrait également disparaître dans les faits - même si de ce point de vue, les bonnes intentions réelles ou supposées se heurtent à de très tenaces résistances.

La division sexuée du travail a constitué un élément central des rapports de genre, et par conséquent des rapports sociaux en général. Elle est intimement liée à l'idée plus globale que les deux sexes devraient nécessairement être assignés à des rôles sociaux spécifiques et, au moins potentiellement, de statut inégal. Même si, d'une société à l'autre, ce mode d'organisation s'est évidemment combiné de mille manières à de nombreux autres facteurs et qu'il ne saurait résumer à lui seul la diversité et la complexité des rapports sociaux, il a toujours et partout représenté une dimension essentielle... jusqu'à notre époque, où il est fortement remis en cause.

Mvts : Doit-on faire une différence entre division sexuelle et division sociale du travail? Si oui, quelle en est la nature : sociale, technique, politique, économique? Si non, faut-il considérer ces deux concepts comme désignant simplement deux extensions différentes du même phénomène?

C. D. : La division sexuelle du travail est simplement une forme particulière de division sociale du travail : celle qui répartit les tâches, comme son nom l'indique, sur la base du sexe.

Elle possède la particularité marquante d'être universelle, et d'être la seule dans ce cas : on a observé des centaines de sociétés de chasseurscueilleurs, et même de petits cultivateurs, où le travail était uniquement divisé en fonction du sexe. Inversement, on n'a jamais vu aucune société où le travail n'était pas réparti en fonction de ce critère, à un degré ou à un autre. On peut donc dire que, sur le plan historique en tout cas, le critère du sexe a constitué la forme première de division sociale du travail.

Ainsi que je le disais, cette division sexuée du travail a perduré dans toutes les sociétés, jusqu'à la nôtre, alors même que, sous ses autres formes, la division sociale du travail (sans parler de sa division technique), inexistante chez les chasseurs-cueilleurs mobiles, s'est depuis considérablement développée.

Mvts : Feriez-vous une distinction entre une division qui opère au sein d'une société où sphère domestique de production et sphère publique ont été séparées, et une division qui opère au sein d'une économie complètement encastrée dans le social ? Pour le dire autrement, la séparation spécifique du domestique et du social, induite par l'émergence de la propriété privée, entraîne-t-elle une mutation de la division sexuelle du travail?

C. D. : Il est bien évident que les modalités par lesquelles les femmes ont été dominées ont beaucoup varié avec les types de société et d'économie. 
À titre de grande généralité, on peut dire que le fil rouge qui traverse ces diverses configurations, c'est l'exclusion des femmes (là encore, plus ou moins marquée et systématique selon les cas) des sphères susceptibles de leur conférer un pouvoir ou, ce qui revient un peu au même, de résister aux pouvoirs masculins. Il est donc clair que dans les économies où la sphère publique s'est démarquée plus ou moins nettement de la sphère domestique, les femmes ont eu tendance à être reléguées dans la seconde. Il faut toutefois se garder de tout simplisme. Contrairement à des idées qui ont longtemps prévalu sur la base d'une méconnaissance des peuples de chasseurs-cueilleurs, la subordination des femmes a été observée dans bien des sociétés où, en matière de travail au moins, l'espace domestique n'était guère distinct de l'espace public. Cette subordination reposait en fait sur leur exclusion des deux grandes sphères de pouvoir qu'étaient le maniement des armes et celui des secrets religieux. Pour les armes, c'est assez évident : le sexe qui les possède est celui qui fait la guerre, qui protège l'autre et qui, par conséquent, se trouve éventuellement en position de le soumettre. Pour la religion, on le sait moins, mais de nombreux peuples de chasseurs-cueilleurs pratiquaient des religions dites à initiations, c'est-à-dire des religions qui comportaient des connaissances, des rites, des sites et des objets dont l'existence était soigneusement dissimulée. Ces secrets étaient révélés uniquement à des adeptes choisis, en l'occurrence les adolescents masculins, après un long apprentissage. De tels cultes représentaient un puissant moyen de contrôle sur les femmes, et malheur à celle qui, même par inadvertance, surprenait un secret ou s'approchait trop d'un site sacré. De telles infractions étaient punies de mort ou de viol collectif.

Mvts: On sait que de nombreux facteurs ont été mis en avant au fil du temps pour expliquer ce phénomène de la division sexuelle du travail (soit en paléoanthropologie et en prébistoire, soit en etbnologie et anthropologie), depuis des déterminismes naturels (caractéristiques physiques) jusqu'à des pratiques culturelles liées à l'écoulement du sang en passant par des déterminismes sociaux liés à la préservation des formes d'organisation familiale de la société. À quoi faut-il attribuer selon vous cette bétérogénéité des modèles explicatifs? Diriez-vous que ceux-ci gardent une certaine pertinence aujourd'bui encore, malgré leur diversité ?

C. D. : Si l'on a tant d'explications différentes sur l'origine de la division sexuée du travail, c'est parce qu'en réalité, on n'en a aucune - aucune qui soit en tout cas suffisamment fondée sur des faits pour emporter l'adhésion.

On est en quelque sorte face à une boîte noire : nous sommes issus d'un monde animal où ce phénomène n'existe pas. Certes, chez les chimpanzés, nos cousins les plus proches, ce sont avant tout les mâles qui chassent. Mais les femelles participent aussi à la chasse et, surtout, il n'existe aucun système de mise en commun des produits entre les sexes. Quant aux quelques outils dont se servent ces singes, ils sont aux mains des femelles! 
4. http://cdarmangeat. blogspot.com/2020/11/ les-femmes-et-lachasse-au.html.

5. Essai sur les fondements de la division sexuelle du travail chez les chasseurs-cueilleurs, Paris, Éditions de l'EHESS, 1986.
La division sexuée du travail au sein de l'espèce humaine s'est donc mise en place au plus tôt à l'époque du dernier ancêtre commun des homo et des chimpanzés et au plus tard durant le Paléolithique supérieur: le fait qu'elle constitue un trait commun à toutes les sociétés humaines observées à l'époque contemporaine rend très improbable l'hypothèse d'une apparition plus récente qui se serait effectuée à la fois de manière indépendante et universelle. À quelques dizaines de millénaires près, la zone d'incertitude temporelle couvre donc à peu près 7 millions d'années, rien que cela!

Et le pire, c'est que les éléments tangibles s'arrêtent à peu près là. Sur cette question, l'archéologie est quasiment muette : plus on remonte dans le temps, plus les restes humains se font rares et fragmentaires. À cela s'ajoute le fait que la division sexuée du travail, hormis dans certaines de ses manifestations assez particulières, ne laisse que fort peu de traces archéologiques, notamment sur les squelettes. Le plus ancien indice sur ce point, à ma connaissance, est une étude menée par S. Vilotte qui tend à montrer que, dès le Paléolithique supérieur, les coudes droits masculins avaient une forte tendance à présenter la même déformation que ceux des lanceurs de base-ball d'aujourd'hui : cela accréditerait donc le scénario d'une division sexuée du travail déjà en place à cette époque, dans laquelle les hommes possédaient le monopole des armes lancées. Le bémol de cette étude est que, par la force des choses, elle porte sur un nombre de squelettes très réduit, et qu'elle n'élimine donc pas formellement la possibilité d'un simple artefact statistique. Quant aux découvertes qui, à grand bruit, proclament de temps à autre qu'elles bouleverseraient ce schéma présenté comme sexiste, elles procèdent généralement d'effets d'annonce qui doivent bien plus à la mauvaise publicité qu'à la bonne science $^{4}$.

À partir de là, on est donc contraint d'échafauder des raisonnements en quelque sorte suspendus en l'air et qui comportent par la force des choses une bonne part de spéculation. Il y a donc eu deux grandes catégories d'explications : selon le "bon sens " traditionnel - qui est aussi celui qui fait dire que la Terre est plate ! - la division sexuelle du travail procéderait de causes physiologiques : la grossesse, puis l'allaitement, empêcheraient la femme de chasser le gros gibier et, par extension, de manier certains outils. Plusieurs chercheurs, dont Alain Testart, ont montré toutes les limites de cette théorie ; je ne détaillerai pas ici leurs arguments mais, en réalité, la division naturelle du travail de reproduction (pour parler de manière un peu pédante) n'explique que très mal, sinon pas du tout, la division sexuée du travail de production.

Les propositions de théories alternatives ont été diverses. Selon Claude Lévi-Strauss, la division du travail avait pour rôle de permettre l'existence de la famille. Selon Testart ${ }^{5}$, elle découlait d'une idéologie qui imposait de séparer le sang des femmes de celui du gibier chassé. En réalité, j’ai bien peur que ces explications ne soient, au mieux, guère plus que des tautologies. La plupart des espèces d'oiseaux vivent en familles monogames sans qu'existe la moindre division sexuée du travail. Et, inversement, la 
division sexuée du travail pourrait fort bien être compatible avec une organisation autre que familiale. Quant à l'idée qu'à l'origine d'une institution sociale aussi fondamentale que la division sexuée du travail, il puisse y avoir une idéologie universelle, elle renvoie immédiatement à la question de savoir d'où, à son tour, proviendrait cette idéologie.

Pour ma part, je crois qu'un bon doute vaut mieux qu'une mauvaise certitude. Il faut accepter d'avouer notre ignorance et de dire franchement que les circonstances et les raisons de l'apparition de la division sexuée du travail nous resteront probablement à jamais inconnues. Un élément tout de même, que l'on connaît depuis Adam Smith au moins, c'est que, d'une manière générale, la division du travail est la clé de l'augmentation de la productivité. Dès lors, quelle que soit l'époque à laquelle cette innovation s'est produite, il est permis de penser qu'elle a assuré aux groupes humains qui l'avaient adoptée un certain avantage dans l'exploitation de leur environnement et dans leur perpétuation. Quant au fait qu'elle ait épousé la ligne de fracture du sexe, il n'est certainement pas étranger à cette base naturelle préexistante - ce qui ne veut absolument pas dire qu'il s'y résume. Les différences physiologiques entre hommes et femmes ont fort bien pu fournir une base de départ dans la construction sociale d'une répartition des tâches, qui s'est creusée au point de dépasser de très loin ses déterminations initiales et de rendre finalement cellesci assez secondaires.

Mvts : Vous vous opposez à plusieurs reprises à l'anti-évolutionnisme qui, depuis Lévi-Strauss, dominerait l'anthropologie. Pourriez-vous développer cet aspect de vos recherches, en particulier autour de deux axes: premièrement, comment l'évolutionnisme se distingue-t-il de la théorie des "stades" qui traverse la théorie économique depuis Smith au moins, et se poursuit jusqu'à la fin du XIX $X^{e}$ siècle, en particulier chez les membres de l'École historique allemande? En d'autres termes, s'agit-il de mettre en évidence des stades universels d'évolution, et si oui, quel rôle joue la division sexuelle du travail dans cette histoire? Deuxièmement, quel rapport entretient cet évolutionnisme aux différentes versions du marxisme?

C. D. : Il faudrait un livre entier pour tenter de répondre correctement à toutes ces questions, et j'ai bien peur que ce livre n'ait encore été écrit par personne! J'ai récemment présenté quelques éléments à ce sujet dans un double article pour la revue Sciences et Pseudo-sciences ${ }^{6}$ auquel je me permets de vous renvoyer.

Pour tenter de dire les choses en quelques mots : il est proprement sidérant que l'anthropologie sociale, en tout cas en France, ait abandonné un des questionnements qui avait toujours été le sien depuis sa fondation, à savoir comment, par quels mécanismes et selon quelles régularités, ou quelles lois, les sociétés évoluent-elles? Je ne dis pas qu'il faut s'accrocher à des réponses dépassées, mais depuis quand, en sciences, de mauvaises réponses rendent-elles illégitime la question qui les a motivées? Au demeurant, les préhistoriens, eux, sont bien obligés de se colleter avec
6. Sciences et Pseudosciences, $\mathrm{n}^{\circ} 335$, février 2021. 
l'évolution sociale, parce qu'elle constitue en quelque sorte la matière première de leur discipline : s'ils veulent comprendre pourquoi les traces matérielles laissées par les sociétés changent, ils sont bien obligés de comprendre comment les sociétés elles-mêmes changent. Et ils ne peuvent le faire qu'avec l'aide d'une anthropologie sociale qui n'aurait pas tourné le dos, par choix idéologique, à cette question scientifique essentielle. Quant au marxisme, c'est évidemment un évolutionnisme - un évolutionnisme qui n'entend pas seulement décrire la manière dont les sociétés se sont transformées par le passé, mais aussi (et surtout !) agir pour transformer de fond en comble la société présente.

Après, la question des stades est éminemment complexe. Elle renvoie en fait au problème de la classification, c'est-à-dire des types de société. Or, contrairement à la biologie, la science des sociétés n'a toujours pas été capable d'élaborer une classification robuste, qui fasse consensus et sur laquelle on puisse raisonner de manière sûre. Pire : personne n'est même d'accord sur les critères pertinents qui permettraient d'y parvenir ! Le marxisme n'échappe pas à ces difficultés : la typologie des modes de production reste en réalité un chantier à peine entamé, et les modes de production ne concernent a priori que les sociétés de classes... Au moins, le marxisme, à défaut d'y apporter une réponse définitive, a-t-il le mérite de ne pas tourner le dos à ces problèmes.

Il faut donc le reconnaître honnêtement : marxistes ou non, nous comprenons et nous formalisons encore assez mal l'objet scientifique complexe qu'est l'évolution des formes sociales humaines. Cela dit, il ne faut pas non plus que l'arbre cache trop la forêt. Plus on entre dans les détails (et il faut y entrer, bien sûr !), plus l'image est bigarrée, pleine d'exceptions et de cas a priori étranges. Mais avec un peu de recul, comme devant un tableau impressionniste, les grandes lignes sont tout de même intelligibles, et la succession des sociétés humaines n'est pas un chaos informe. Les "stades" constituent certes un outil conceptuel grossier, qui pose de multiples problèmes et qu'il faut s'efforcer d'affûter. Mais un outil grossier, c'est tout de même nettement mieux que pas d'outil du tout, et les seuls qui réussissent vraiment à se passer des "stades ", quels qu'ils soient, ce sont ceux qui refusent d'étudier l'évolution sociale.

Pour finir sur un aspect plus précis, je suis bien incapable de dire quel rôle la division sexuée du travail a pu jouer dans tout cela. Mon intuition me suggère qu'elle n'est intervenue tout au plus que sur des aspects relativement secondaires. Pour ne parler que des sujets sur lesquels j'ai travaillé, je n'ai pas l'impression, par exemple, qu'elle ait pesé d'un poids particulier dans l'émergence de la richesse ou, plus tard, dans celle des classes sociales. Mais les phénomènes sociaux sont toujours d'une grande complexité, il n'est pas du tout impossible qu'on réalise un jour que telle modalité de la division sexuée du travail a pu être impliquée dans telle ou telle bifurcation des structures sociales. 
travail de ses effets politiques. La séparation fonctionnelle des tâches ne veut pas dire, empiriquement et automatiquement, domination masculine. Pouvez-vous expliquer cette thèse?

C. D. : Le fait d'assigner des tâches différentes aux hommes et aux femmes est effectivement une condition nécessaire, mais non suffisante, de la domination des premiers sur les secondes. Pour prendre un exemple trivial, si, dans un couple, elle fait le bricolage et lui la vaisselle (ou l'inverse), cela ne préjuge pas, en soi, de rapports de pouvoir et d'une iniquité du partage. Par contre, il ne saurait y avoir de domination sociale sans une division des tâches qui assure à l'un des deux une position dominante sur l'autre.

Au passage, cette position dominante s'est bien davantage construite, me semble-t-il, comme une conséquence du fait que les hommes détenaient les moyens matériels de la violence, que sur la base de l'exploitation économique des femmes. Les hommes, dans les sociétés de chasseurs-cueilleurs puis de petits cultivateurs, dominent les femmes parfois très durement, mais ne sont pas des rois fainéants. Même si les données ethnologiques restent fragmentaires et difficiles à interpréter, elles n'indiquent pas qu'ils mettent leur avantage à profit pour travailler moins qu'elles. Ce phénomène ne semble s'être développé que dans un second temps : lorsque la richesse a émergé et a pénétré les rapports sociaux, les dominées ont eu tendance à devenir de surcroît des exploitées.

Un autre aspect, beaucoup plus actuel, de la question, c'est que le " respect des différences " des sexes - traduisez, l'assignation de chaque sexe à des tâches et des rôles spécifiques - est devenu sous nos latitudes le faux nez de ceux qui militent pour que les femmes restent à leur place (inférieure), sans pouvoir le revendiquer ouvertement. C'était tout à fait perceptible dans les slogans de la "Manif pour tous ", il y a quelques années. Une telle revendication, avec tout ce qu'elle peut comporter d'hypocrisie, est un peu le remake sexiste du "séparés, mais égaux " de la ségrégation raciale. Il va de soi que l'égalité des sexes du présent et de l'avenir ne pourra se construire sur la base de la perpétuation de la division sexuée des tâches, mais au contraire sur sa disparition. Les configurations où régnaient à la fois une division sexuée des tâches et un certain équilibre entre les sexes, qu'on qualifie parfois, par abus de langage, de "matriarcats ", sont certes intéressantes du point de vue historique et scientifique. Il est cependant clair qu'il s'agit d'un modèle de société qui appartient au passé. Et bien que certains courants féministes l'idéalisent volontiers, je ne suis pas du tout certain qu'il faille en éprouver quelque nostalgie que ce soit.

Mvts : Si seules certaines des sociétés sans classes relativement égalitaires ont été épargnées par la domination masculine, là où, au contraire, les sociétés de classes l'ont toujours connue, comment situer cette dernière au sein du devenir capitaliste des sociétés contemporaines? Quels enseignements politiques (notamment en termes de stratégies militantes et de lutte) peut-on en retirer selon vous? 
C. D. : Les données ethnologiques montrent sans contestation possible qu'historiquement - ou, si l'on préfère, préhistoriquement ! - la domination masculine a précédé, de beaucoup, la domination de classe. Dans bien des tribus d'Australie aborigène, ou chez les Indiens de la Terre de Feu, et même chez certains peuples de Nouvelle-Guinée, il n'y a pas le début d'une différenciation par la richesse ; pourtant, il règne une domination masculine parfois féroce.

Le développement des inégalités de richesse, prélude à la structuration en classes, joue à cet égard un rôle ambivalent. Dans bien des circonstances, en incitant les hommes à exploiter le travail des femmes, elle leur fournit à la fois un motif et un moyen supplémentaires de les dominer. Mais, en même temps, elle contribue au moins dans certains cas au relâchement de formes de contrôle plus directes. En Nouvelle-Guinée, par exemple, les sociétés à richesse sont aussi celles qui sont dépourvues des grandes initiations masculines et des formes les plus pesantes de subordination des femmes. Toute la difficulté, c'est qu'il existe des centaines de configurations possibles, et qu'il est donc très hasardeux d'isoler l'action d'un seul facteur qui, dans la réalité, se combine toujours avec des dizaines d'autres. En tout cas, il n'est pas si évident que, d'une manière globale, l'émergence des inégalités de richesse ait nécessairement aggravé le sort des femmes. Il est difficile de se prononcer à ce niveau de généralité. Mais, au passage, la plupart des exemples de "matriarcats " régulièrement invoqués, au-delà du caractère abusif de cette dénomination, sont des sociétés dans lesquelles la richesse existait !

Quant au fait que les femmes aient systématiquement été infériorisées dans les sociétés de classe, je pense que le constat est indéniable, mais ne suis pas certain de la manière dont il s'explique. Pour commencer, ce phénomène n'est-il pas la simple conséquence probabiliste de la fréquence de la domination masculine, et de la rareté relative des situations où les femmes ont été en mesure de la contrecarrer? Quoi qu'il en soit, j'aurais tendance à penser que la structuration en classes n'est pas en elle-même un facteur d'aggravation de la domination masculine. Je suggérerais volontiers que, dans certains endroits du monde au moins, c'est un même phénomène technique - la mise en place de l'agriculture attelée - qui a à la fois sapé certaines positions économiques des femmes et permis l'émergence des classes et des États. En tout état de cause, il me semble que ces phénomènes sont encore assez mal compris, sinon mal connus.

Enfin, que le capitalisme non seulement puisse s'accommoder d'une relative égalité des sexes mais, que dans une certaine mesure et dans certaines circonstances, il la promeuve activement, c'est un fait qui ne peut guère, je crois, être contesté. Nous vivons dans des sociétés dont, depuis des décennies, les dirigeants se sont sentis obligés de défendre "l'égalité des sexes " (c'est-à-dire leur identité sociale), en levant notamment, les unes après les autres, les dispositions légales qui infériorisaient les femmes. Bien sûr, tout cela ne s'est fait ni tout seul ni en un jour. Une partie de ces conquêtes - en particulier, autour de l'IVG - est éminemment 
fragile et doit encore être vigoureusement défendue contre les menaces de régressions. Cependant, la perspective d'un retour complet de nos sociétés à une situation évoquant le Code Napoléon, à la manière décrite dans la dystopie La Servante écarlate, si elle n'est pas totalement impossible, semble largement improbable. À grande échelle, spatiale et historique, le mouvement porté par le capitalisme vers l'égalité (au moins juridique) des individus suscite globalement une évolution qui tend à lever peu à peu les discriminations contre les femmes, et non à les renforcer. Certes, l'égalité juridique n'est pas l'égalité réelle et, sur bien des plans, on peut même dire que la domination masculine de facto résiste très bien à sa disparition de jure. Je crois néanmoins que même s'il reste beaucoup à faire et même si, une fois encore, il est dangereux de s'exprimer par généralités, il n'est guère contestable que l'égalité juridique qui s'est peu à peu imposée dans le dernier siècle est allée de pair avec une certaine émancipation pour les femmes.

Après, il y a la question de savoir pour quel modèle de société on doit agir, et en s'appuyant sur quelle force sociale. Cette question est un peu différente de celle de l'analyse et du constat même si, évidemment, elles ne sont pas sans rapport. Au risque de choquer, je n'ai jamais très bien compris ce qu'apportait la perspective dite intersectionnelle. S'il s'agit de constater que les individus sont pris dans des déterminations multiples et que la position sociale ne se résume pas à la classe (fondamentale), il me semble que c'est une évidence depuis toujours, en particulier pour les militants, qui se confrontent au quotidien à ces réalités. J'ai néanmoins le sentiment que, le plus souvent, l'insistance placée sur ce point aboutit à reléguer au second plan la lutte des classes et, en réalité, à l'abandonner purement et simplement.

Pour finir, je crois que, pour diverses raisons, on peut légitimement douter de la possibilité de parvenir à une égalité des sexes pleine et entière au sein de la société capitaliste. Mais, à la limite, c'est une question qui ne peut intéresser que les personnes qui, tout en menant le combat féministe, ne veulent pas remettre en cause la domination du capital sur la société. Pour celles et ceux qui entendent lutter contre toutes les oppressions, quelles qu'elles soient, elle est en quelque sorte sans objet, car purement théorique : les deux luttes doivent être menées conjointement. On peut d'ailleurs rappeler qu'avant de tomber dans l'atonie qui est hélas la sienne depuis des décennies, le mouvement ouvrier alors révolutionnaire était à l'avant-garde du combat féministe. C'est par exemple à son initiative que fut créée la journée du 8 mars et, en matière familiale, la Russie de 1917 avait mis en place la législation de loin la plus avancée de son époque. 